Preprint JINR E2-98-303

\title{
On a different BRST constructions for a given Lie algebra
}

\author{
A. Pashnev \\ and M. Tsulaiai \\ JINR-Bogoliubov Theoretical Laboratory, \\ 141980 Dubna, Moscow Region, Russia
}

\begin{abstract}
The method of the BRST quantization is considered for the system of constraints, which form a Lie algebra. When some of the Cartan generators do not imply any conditions on the physical states, the system contains the first and the second class constraints. After the introduction auxiliary bosonic degrees of freedom for these cases, the corresponding BRST charges with the nontrivial structure of nonlinear terms in ghosts are constructed.

Submitted to Proceedings of the Conference "Supersymmetry and Quantum Symmetries" dedicated to the memory of V.I. Ogievetsky
\end{abstract}

\footnotetext{
*E-mail: pashnev@thsun1.jinr.dubna.su

${ }^{\dagger}$ E-mail: tsulaia@thsun1.jinr.dubna.su
} 


\section{Introduction}

The BRST quantization procedure for a system of the first class constraints is straightforward. By the definition, the first class constraints form a closed algebra with respect to the commutators (the Poisson brackets). For simplicity we consider only linear algebras - Lie algebras of constraints.

More general systems include the second class constraints as well, whose commutators contain terms which are nonzero on mass shell (on the subspace where all constraints vanish). In the simplest cases these terms are a numbers or central charges, but sometimes, they are operators which act nontrivially on the space of the physical states. Moreover, the commutators between these operators and the constraints can be nontrivial. In some cases the total system of the constraints and the operators mentioned above form a Lie algebra.

So, in such cases we have a system of operators which form a Lie algebra, but the physical meaning of different operators is different. Some of them play the role of constraints and annihilate the physical states, others are nonzero and simply transform the physical states into other ones. It means, that in the BRST approach for the description of the corresponding physical system we can not use the standard BRST charge for the given Lie algebra. Instead, we have to construct the nilpotent BRST charge in a manner, that some of the operators play the role of the first class constraints, others are second class constraints and the others do not imply any conditions on the physical space of the system.

In this paper we demonstrate the possibility of a different BRST constructions for the system of generators, which form a given Lie algebra and have different physical meaning. In the second part we describe some algebraic approaches, leading to the description of massless (or massive) irreducible representations of the Poincare group in any dimensions. As the simplest example we show, that the same algebra of operators leads to either massless, or massive spectra in the cases when physical meaning of some operators is different. In the third part we discuss the general method of the BRST quantization, when some of the Cartan generators are excluded from the total system of constraints. In the fourth part we describe the construction of auxiliary representations of the algebra by means of the Gel'fand-Tsetlin method. In the Section 5 we give the simple example.

\section{The description of constraints}

In order to kill ghosts the field theoretical lagrangians, describing irreducible Poincare representations must possess some gauge invariance. Along with the basic fields such lagrangians in general include additional ones. The role 
of these fields is to single out the irreducible representation of the Poincare group. Some of them are auxiliary, others can be gauged away. After a gauge fixing and solving the equations of motion for auxiliary fields one is left with the only essential field, describing the irreducible representation of the Poincare group. This field corresponds to the Young tableaux with $k$ rows

\begin{tabular}{|c|c|c|c|c|c|c|c|}
\hline \begin{tabular}{|l|l|}
$\mu_{1}$ & $\mu_{2}$ \\
\end{tabular} & & . & . & . & . & & $\cdot \mu_{n}$ \\
\hline \begin{tabular}{|l|l|}
$\nu_{1}$ & $\nu_{2}$ \\
\end{tabular} & & . & . & . & . & $\nu_{n_{2}}$ & \\
\hline . & & . & . & . & . & & \\
\hline \begin{tabular}{|l|l|}
$\rho_{1}$ & $\rho_{2}$ \\
\end{tabular} & & $\rho_{n_{k}}$ & & & & & \\
\hline
\end{tabular}

and is described by $\Phi_{\mu_{1} \mu_{2} \cdots \mu_{n_{1}}, \nu_{1} \nu_{2} \cdots \nu_{n}}^{(k) \cdots, \rho_{1} \rho_{2} \cdots \rho_{n_{k}}}(x)$ which is the $n_{1}+n_{2}+\cdots+$ $n_{k}$ rank tensor field symmetrical with respect to the permutations of each type of indices. In addition, this field is subject to the following system of equations, namely the mass shell and transversality conditions for each type of indices. In the massless case we have

$$
\begin{aligned}
& p_{\mu}^{2} \Phi_{\mu_{1} \mu_{2} \cdots \mu_{n_{1}}, \nu_{1} \nu_{2} \cdots \nu_{n_{2}}, \cdots, \rho_{1} \rho_{2} \cdots \rho_{n_{k}}}^{(k)}(x)=0, \\
& p_{\mu} \Phi_{\mu \mu_{2} \cdots \mu_{n_{1}}, \nu_{1} \nu_{2} \cdots \nu_{n_{2}}, \cdots, \rho_{1} \rho_{2} \cdots \rho_{n_{k}}}^{(k)}(x)=0, \\
& p_{\rho} \Phi_{\mu_{1} \mu_{2} \cdots \mu_{n_{1}}, \nu_{1} \nu_{2} \cdots \nu_{n_{2}}, \cdots, \rho \rho_{2} \cdots \rho_{n_{k}}}^{(k)}(x)=0 .
\end{aligned}
$$

Further, all traces of the basic field must vanish:

$$
\begin{aligned}
& \Phi_{\mu \mu \mu_{3} \cdots \mu_{n_{1}}, \nu_{1} \nu_{2} \cdots \nu_{n_{2}}, \cdots, \rho_{1} \rho_{2} \cdots \rho_{n_{k}}}^{(k)}(x)=0, \\
& \Phi_{\mu \mu_{2} \mu_{3} \cdots \mu_{n_{1}}, \mu \nu_{2} \cdots \nu_{n_{2}}, \cdots, \rho_{1} \rho_{2} \cdots \rho_{n_{k}}}^{(k)}(x)=0, \\
& \cdots \cdots \cdots \cdots \cdots \cdots \cdots \cdots \cdots \cdots \cdots \cdots \cdots \cdots \cdots \cdots \\
& \Phi_{\mu_{1} \mu_{2} \cdots \mu_{n_{1}}, \nu_{1} \nu_{2} \cdots \nu_{n_{2}}, \cdots, \rho \rho \rho_{3} \cdots \rho_{n_{k}}}^{(k)}(x)=0 .
\end{aligned}
$$

The correspondence with a given Young tableaux implies, that after symmetrization of all indices of one type with one index of another type, the basic field vanishes, for example

$$
\Phi_{\left\{\mu_{1} \mu_{2} \cdots \mu_{\mathbf{n}_{\mathbf{1}}}, \nu_{\mathbf{1}}\right\} \nu_{\mathbf{2}} \cdots \nu_{\mathbf{n}_{2}}, \cdots, \rho_{\mathbf{1}} \rho_{\mathbf{2}} \cdots \rho_{\mathbf{n}_{\mathbf{k}}}}^{(k)}(x)=0 .
$$

To describe all irreducible representations of the Poincare group simultaneously it is convenient to introduce an auxiliary Fock space generated by the creation and annihilation operators $a_{\mu}^{i+}, a_{\mu}^{j}$ with Lorentz index $\mu=0,1,2, \ldots, D-$ 1 and additional internal index $i=1,2, \ldots, k$. These operators satisfy the following commutation relations

$$
\left[a_{\mu}^{i}, a_{\nu}^{j+}\right]=-g_{\mu \nu} \delta^{i j}, g_{\mu \nu}=\operatorname{diag}(1,-1,-1, \ldots,-1)
$$


where $\delta^{i j}$ is usual Cronecker symbol.

The general state of the Fock space depends on the space-time coordinates $x_{\mu}$

$$
\begin{aligned}
|\Phi\rangle= & \sum \Phi_{\mu_{1} \mu_{2} \cdots \mu_{n_{1}}, \nu_{1} \nu_{2} \cdots \nu_{n_{2}}, \cdots, \rho_{1} \rho_{2} \cdots \rho_{n_{k}}}^{(k)}(x) \times \\
& a_{\mu_{1}}^{1+} a_{\mu_{2}}^{1+} \cdots a_{\mu_{n_{1}}}^{1+} a_{\nu_{1}}^{2+} a_{\nu_{2}}^{2+} \cdots a_{\nu_{n_{2}}}^{2+} \cdots a_{\rho_{1}}^{k+} a_{\rho_{2}}^{k+} \cdots a_{\rho_{n_{k}}}^{k+}|0\rangle
\end{aligned}
$$

and the components $\Phi_{\mu_{1} \mu_{2} \cdots \mu_{n_{1}}, \nu_{1} \nu_{2} \cdots \nu_{n_{2}}, \cdots, \rho_{1} \rho_{2} \cdots \rho_{n_{k}}}^{(k)}(x)$ are automatically symmetrical under the permutations of indices of the same type [1] - [3]. The norm of states in this Fock space is not positively definite due to the minus sign in the commutation relation (2.1) for the time components of the creation and annihilation operators. The transversality conditions (2.3)-(2.4) for the components are equivalent to the following constraints on the physical vectors of the Fock space

$$
L^{i}|\Phi\rangle=0
$$

where

$$
L^{i}=a_{\mu}^{i} p_{\mu}
$$

These operators along with their conjugates

$$
L^{i+}=a_{\mu}^{i+} p_{\mu}
$$

and mass shell operator $p_{\mu}^{2}$ form the following algebra with only nonvanishing commutator

$$
\left[L^{i}, L^{j+}\right]=-p_{\mu}^{2} \delta^{i j}
$$

This simple algebra was considered in [1] in the framework of the BRST approach. The constraints are of the first class and nilpotent BRST charge can be constructed without problems. As a result the description of mixed symmetry fields was obtained. However, all these fields describe the reducible representations of the Poincare group due to the absence of additional conditions (2.5)-(2.7) and (2.8) in the initial system of the constraints.

On the other hand, the same algebra of operators arises in the case of massive particles. The only difference is that the right hand side of the relation (2.14) is now nonvanishing operator. Instead, this operator can have different eigenvalues $p_{\mu}^{2}=m_{n}^{2}$ for the different physical states. This situation was analyzed in [1], where corresponding BRST charge, obviously different from the one for the massless case, was constructed using the method of dimensional reduction. As an artifact of this method the construction automatically includes some additional auxiliary variables. So, (2.14) produces the nontrivial example for the different BRST constructions, corresponding to different physical meaning of the generators $\left(p_{\mu}^{2}\right.$ in our case). In what follows we describe the method of alternative constructions of BRST charges which is valid not only for a simple algebras like (2.14). 
The tracelessness conditions (2.5)-(2.7) correspond in the Fock space to the constraints

$$
L^{i j}|\Phi\rangle=0
$$

with

$$
L^{i j}=a_{\mu}^{i} a_{\mu}^{j}, \quad L^{i j+}=a_{\mu}^{j+} a_{\mu}^{i+} .
$$

while the symmetry properties (2.8) follow from the constraints

$$
T^{i j}|\Phi\rangle=0, \quad i<j,
$$

having the explicit form

$$
T^{i j}=a_{\mu}^{i+} a_{\mu}^{j}, \quad T^{i j+}=a_{\mu}^{j+} a_{\mu}^{i}=T_{j i} .
$$

The operators $L^{i j}, L^{i j+}\left(i, j\right.$ are arbitrary) and $T^{i j},(i \neq j)$, along with the additional operators

$$
H^{i}=-T^{i i}+\frac{D}{2}=-a_{\mu}^{i+} a_{\mu}^{i}+\frac{D}{2},
$$

form the Lie algebra $S O(k+1, k)$. The rank of this algebra is $k$ and corresponding Cartan subalgebra contains all operators $H^{i}$. One can choose the operators $L^{11}$ and $T^{i, i+1}$ as $k$ simple roots. The positive and negative roots are, correspondingly, $L^{i j}, T^{r s},(1 \leq r<s \leq k)$ and $L^{i j+}, T^{r s},(1 \leq s<$ $r \leq k)$. It means, that the conditions (2.5)-(2.7) and (2.8) are equivalent to annihilation of physical states in the total Fock space by the positive roots of the Lie algebra $S O(k+1, k)$.

As it can be easily seen, the Cartan generators (2.19) are strictly positive in the Fock space and therefore the standard BRST charge has to be modified for the given realization of the $S O(k+1, k)$ algebra.

The BRST approach to the construction of the lagrangians, from which all the equations (2.2)-(2.8) follow, is very powerful. It automatically leads to appearance of all auxiliary fields in the lagrangian. In the massless case the BRST charge for the system of only first class constraints, corresponding to the equations (2.2) - (2.4) was constructed in [四].

The methods of such construction were discussed in [5] - [7]. With the help of additional variables one can modify the second class constraints in such a way that they become commuting, i.e. the first class. At the same time the number of physical degrees of freedom for both systems does not change if the number of additional variables coincides with the number of second class constraints.

On the other hand, the BRST charge for the second class constraints in some cases can be constructed using the method of dimensional reduction. In [4] the system of massive higher spins satisfying equations

$$
\left(p_{\mu}^{2}-m^{2}\right) \Phi_{\mu_{1} \mu_{2} \cdots \mu_{n_{1}}}(x)=0
$$


and

$$
p_{\mu} \Phi_{\mu \mu_{2} \cdots \mu_{n_{1}}}(x)=0
$$

was described in the framework of the BRST approach. From the point of view of $D$ dimensions, where constraint (2.21) is of second class, the $D+1$-st components of the creation and annihilation operators appear in the consideration as additional operators. The corresponding BRST charge is nilpotent and has a very special structure. In particular, the modified constraints have the algebra, which is not closed. Nevertheless, the nontrivial structure of trilinear terms in ghosts in the BRST charge compensates this defect and makes the BRST charge to be nilpotent. Another example of BRST charge for the system, including second class constraints was obtained in [8]. It reproduces some properties of the BRST charge of [4]: the algebra of modified constraints is not closed and trilinear terms in ghosts are nontrivial as well. Moreover, the BRST charge contains terms up to the seventh degree in ghosts. In the next Section we will describe the simple method, which allows to one to construct the nilpotent BRST charges for a given Lie algebra, when some generators are treated as the second class constraints. As a particular case, this method reproduces the results obtained in [4] and [8].

\section{The general method}

In this section, we describe the method of the BRST construction, which leads to the desirable division of the generators of a given Lie algebra into the first and second class constraints. Let $H^{i}, \quad(i=1, \ldots, k)$ and $E^{\alpha}$ be the Cartan generators and root vectors of the algebra with the following commutation relations

$$
\begin{aligned}
& {\left[H^{i}, E^{\alpha}\right]=\alpha(i) E^{\alpha},} \\
& {\left[E^{\alpha}, E^{-\alpha}\right]=\alpha^{i} H^{i},} \\
& {\left[E^{\alpha}, E^{\beta}\right]=N^{\alpha \beta} E^{\alpha+\beta} .}
\end{aligned}
$$

Roots $\alpha(i)$ and parameters $\alpha^{i}, N^{\alpha \beta}$ are structure constants of the algebra in the Cartan - Weyl basis. Our goal is to construct nilpotent BRST charge, which after quantization leads to the following conditions: all positive root vectors $E^{\alpha}(\alpha>0)$ of the algebra annihilate the physical states. Contrary, the operators $H^{i}$ which form the Cartan subalgebra may or may not be constraints, depending on the physical nature of these operators.

The simplest case, when all Cartan generators annihilate the physical states, is well known. We introduce the set of anticommuting variables $\eta_{i}, \eta_{\alpha}$, $\eta_{-\alpha}=\eta_{\alpha}^{+}$, having ghost number one and corresponding momenta $\mathcal{P}_{i}, \mathcal{P}_{-\alpha}=$ 
$\mathcal{P}_{\alpha}^{+}, \mathcal{P}_{\alpha}$, with the commutation relations:

$$
\left\{\eta_{i}, \mathcal{P}_{k}\right\}=\delta_{i k},\left\{\eta_{\alpha}, \mathcal{P}_{-\beta}\right\}=\left\{\eta_{-\alpha}, \mathcal{P}_{\beta}\right\}=\delta_{\alpha \beta}
$$

we define the "ghost vacuum" as

$$
\eta_{\alpha}|0\rangle=\mathcal{P}_{\alpha}|0\rangle=\mathcal{P}_{i}|0\rangle=0
$$

for positive roots $\alpha$. The BRST charge for the Cartan - Weyl decomposition of the algebra has a standard form

$$
\begin{aligned}
Q= & \sum_{i} \eta_{i} H^{i}+\sum_{\alpha>0}\left(\eta_{\alpha} E^{-\alpha}+\eta_{-\alpha} E^{\alpha}\right)-\frac{1}{2} \sum_{\alpha \beta} N^{\alpha \beta} \eta_{-\alpha} \eta_{-\beta} \mathcal{P}_{\alpha+\beta}+ \\
& \sum_{\alpha>0, i}\left\{\alpha(i)\left(\eta_{i} \eta_{\alpha} \mathcal{P}_{-\alpha}-\eta_{i} \eta_{-\alpha} \mathcal{P}_{\alpha}\right)+\alpha^{i} \eta_{\alpha} \eta_{-\alpha} \mathcal{P}_{i}\right\}
\end{aligned}
$$

The physical states are then the cohomology classes of the BRST operator.

The quantization in this case is equivalent to the quantization à la Gupta - Bleuler, because physical states satisfy equations $H^{i}|P h y s\rangle=0$ and $E^{\alpha} \mid$ Phys $\rangle=0$ only for positive values of $\alpha$.

The situation changes when some of the Cartan operators $H^{i}$, say $H^{i_{l}}, l=$ $1,2, \ldots N$ are nonvanishing from the physical reasons. In this case the following method can be used.

First of all we construct some auxiliary representation for the generators $H^{i}, E^{\alpha}$ of the algebra in terms of additional creation and annihilation operators. The only condition for this representation is that it depends on some parameters $h^{n}$. The total number of these parameters is equal to the number of the Cartan generators, which are nonzero in the physical sector. In what follows, we consider the realizations of the algebra with a linear dependence of the Cartan generators on these parameters: $\hat{H}^{m}(h)=\tilde{H}^{m}+c_{n}^{m} h^{n}$, where $c_{n}^{m}$ are some constants. The $h^{n}$ dependence of other generators can be arbitrary. In the next section we describe the general method of construction of such representations. Here we simply assume that they exist.

The next step is to consider the realization of the algebra as a sum of "old" and "new" generators

$$
\mathcal{H}^{i}=H^{i}+\hat{H}^{i}(h), \quad \mathcal{E}^{\alpha}=E^{\alpha}+\hat{E}^{\alpha}(h) .
$$

The BRST charge for the total system has the same form as (3.6), with modified generators:

$$
\begin{aligned}
\mathcal{Q}= & \sum_{i} \eta_{i} \mathcal{H}^{i}+\sum_{\alpha>0}\left(\eta_{\alpha} \mathcal{E}^{-\alpha}+\eta_{-\alpha} \mathcal{E}^{\alpha}\right)-\frac{1}{2} \sum_{\alpha \beta} N^{\alpha \beta} \eta_{-\alpha} \eta_{-\beta} \mathcal{P}_{\alpha+\beta}+ \\
& \sum_{\alpha>0, i}\left\{\alpha(i)\left(\eta_{i} \eta_{\alpha} \mathcal{P}_{-\alpha}-\eta_{i} \eta_{-\alpha} \mathcal{P}_{\alpha}\right)+\alpha^{i} \eta_{\alpha} \eta_{-\alpha} \mathcal{P}_{i}\right\}
\end{aligned}
$$


The ghost variables $\eta_{i_{l}}$, correspond to the set of nonvanishing generators $H^{i_{l}}$ and therefore one needs to remove the $\eta_{i_{l}}$ dependence

$$
Q_{i_{l}}=\eta_{i_{l}}\left\{H^{i_{l}}+\tilde{H}^{i_{l}}+c_{n}^{i_{l}} h^{n}+\sum_{\beta>0} \alpha\left(i_{l}\right)\left(\eta_{\beta} \mathcal{P}_{-\beta}-\eta_{-\beta} \mathcal{P}_{\beta}\right)\right\}
$$

from the BRST charge. For this purpose consider an auxiliary $N$ - dimensional space with coordinates $x_{i_{l}}$ and conjugated momenta $p^{i_{l}}$, where $c_{n}^{i_{l}} h^{n}=p^{i_{l}}$ :

$$
\left[x_{i_{l}}, p^{i_{n}}\right]=i \delta_{i_{l}}^{i_{n}}
$$

After the similarity transformation, which corresponds to the dimensional reduction [四]

$$
\tilde{\mathcal{Q}}=e^{i \pi^{i} l x_{i_{l}}} Q e^{-i \pi^{i} l x_{i_{l}}}
$$

where

$$
\pi^{i_{l}}=H^{i_{l}}+\tilde{H}^{i_{l}}++\sum_{\beta>0} \alpha\left(i_{l}\right)\left(\eta_{\beta} \mathcal{P}_{-\beta}-\eta_{-\beta} \mathcal{P}_{\beta}\right)
$$

the transformed BRST charge $\tilde{\mathcal{Q}}$ does not depend on the ghost variables $\eta_{i_{l}}$. All parameters $p^{i_{l}}$ in the BRST charge are replaced by the corresponding operators $\pi^{i_{l}}$. The transformation (3.10) does not change the nilpotency property of the BRST charge. It means that the $\mathcal{P}_{i_{l}}$ independent part $\tilde{\mathcal{Q}}_{0}$ of the total charge $\tilde{\mathcal{Q}}$ is nilpotent as well. Moreover, as a consequence of the nilpotency of $\tilde{\mathcal{Q}}$ all coefficients at the corresponding antighost operators $\mathcal{P}_{i_{l}}$

commute with $\tilde{\mathcal{Q}}_{0}$. One can show that the quantization with the help of the BRST operator $\tilde{\mathcal{Q}}_{0}$ will lead to the desirable reduced system of constraints on the physical states.

\section{Construction of auxiliary representations of the algebra}

Consider the highest weight representation of the algebra under consideration with the highest weight vector $|\Phi\rangle$ annihilated by the positive roots

$$
E^{\alpha}|\Phi\rangle=0
$$

and being the proper vector of the Cartan generators

$$
H^{i}|\Phi\rangle=h^{i}|\Phi\rangle
$$

As it was shown by Gelfand and Tsetlin [9], each of the vectors of the irreducible representation with a given highest weight can be associated with the so called Gelfand-Tsetlin scheme. Corresponding scheme for $U(k)$ algebra has the following form: 


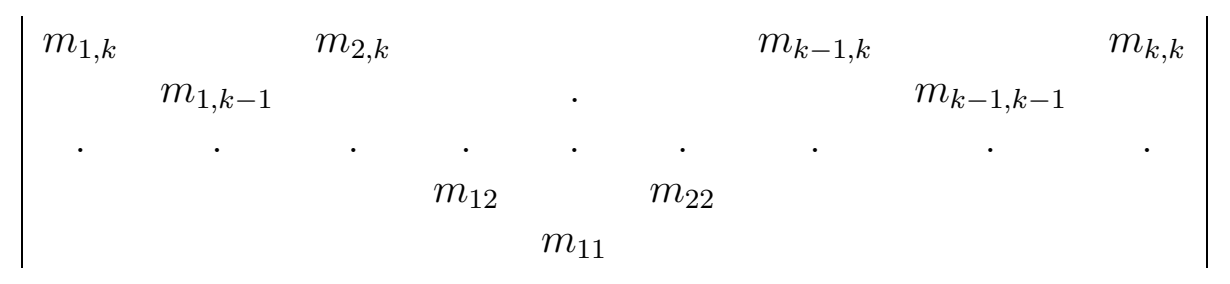

The first row of the scheme is defined by the highest weight components:

$$
m_{i, k}=h^{i} .
$$

This row is fixed for a given irreducible representation of $U(k)$. All other rows contain arbitrary numbers under the following conditions:

$$
m_{i j} \geq m_{i, j-1} \geq m_{i+1, j}, j=2, \ldots, k ; i=1, \ldots, k-1 .
$$

Any choice of this numbers, consistent with (4.4), corresponds to a fixed vector in the irreducible representation. All these vectors are orthonormal. The total number of $m_{i, j}$ coincides with the number of positive roots of the algebra $U(k)$. This is true for any semisimple algebra. It means, that all such vectors can be represented in an auxiliary Fock space, generated by the oscillators $b_{i, j}, b_{i, j}^{+}$, which are in one to one correspondence with numbers $m_{i, j}$. So the vector, which corresponds to the Gelfand - Tsetlin scheme given above has the following form

$$
\left|\left\{m_{i, j}\right\}\right\rangle=\prod_{i, j} \frac{1}{\left(m_{i, j}-\kappa_{i, j}\right)^{1 / 2}}\left(b_{i, j}^{+}\right)^{\left(m_{i, j}-\kappa_{i, j}\right)}|0\rangle,
$$

where $\kappa_{i, j}$ are some fixed parameters, connected with the weights $h^{i}$. Having at hands all matrix elements of generators [9] one can easily reconstruct the expressions for the generators in terms of oscillators $b_{i, j}, b_{i, j}^{+}$. They will depend on $k$ parameters $h^{i}$ and give the needed auxiliary representation of the algebra. Below we construct such representation for the simplest example of $S O(2,1)$ to illustrate the method of the BRST construction described in the previous section.

\section{The simple example}

In this section we consider $S O(2,1)$ algebra of constraints. The Fock space introduced in the Section 2 is spanned by only one oscillator. This system describes higher spin irreducible representations of the Poincare algebra cor-

responding to the Young tableaux with only one row. The total system of generators includes

$$
\begin{aligned}
& L_{1}=a_{\mu} p_{\mu}, \quad L_{-1}=a_{\mu}^{+} p_{\mu}, \quad L_{0}=p_{\mu}^{2}, \quad\left[L_{1}, L_{-1}\right]=-L_{0}, \\
& L_{2}=a_{\mu} a_{\mu}, \quad L_{-2}=a_{\mu}^{+} a_{\mu}^{+}, \quad\left[L_{2}, L_{-2}\right]=-a_{\mu}^{+} a_{\mu}-\frac{D}{2} \equiv G_{0},
\end{aligned}
$$


where $D$ is the dimensionality of the space - time. The last three operators $L_{2}, L_{-2}$ and $G_{0}$ form an $S O(2,1)$ algebra. The first three operators in (5.1) transform as a representation of this algebra and they can be included in the BRST charge rather trivially. So, the main problem is to construct the BRST charge for $S O(2,1)$ algebra under the condition, that the operator $G_{0}$ is not a constraint in the physical subspace, since $G_{0}$ is positively definite in the whole Fock space.

Using the results of the previous Section one can easily construct the auxiliary representation of the algebra $S O(2,1)$ in terms of one additional timelike oscillator $\left[b, b^{+}\right]=-1$ :

$$
\begin{aligned}
\hat{L}_{2} & =\sqrt{h+b^{+} b} b \\
\hat{L}_{-2} & =b^{+} \sqrt{h+b^{+} b} \\
\hat{G}_{0} & =\tilde{G}_{0}-h, \quad \tilde{G}_{0}=-2 b^{+} b .
\end{aligned}
$$

The BRST charge for modified generators

$$
\mathcal{L}_{2}=L_{2}+\hat{L}_{2}, \quad \mathcal{L}_{-2}=L_{-2}+\hat{L}_{-2}, \quad \mathcal{G}_{0}=G_{0}+\hat{G}_{0}
$$

takes the following form

$$
\mathcal{Q}=\eta_{0} \mathcal{G}_{0}+\eta_{2} \mathcal{L}_{-2}+\eta_{-2} \mathcal{L}_{2}-2 \eta_{0} \eta_{2} \mathcal{P}_{-2}+2 \eta_{0} \eta_{-2} \mathcal{P}_{2}+\eta_{2} \eta_{-2} \mathcal{P}_{0}
$$

As the result of similarity transformation described in the Section 3, the coefficient at ghost variable $\eta_{0}$ vanishes and the following replacement of parameter $h$ takes place:

$$
h \rightarrow G_{0}-2 b^{+} b+2 \mathcal{P}_{-2} \eta_{2}+2 \eta_{-2} \mathcal{P}_{2}
$$

The resulting nilpotent BRST charge after removing of the antighost variable $\mathcal{P}_{0}$ looks as follows

$$
\begin{aligned}
\mathcal{Q}_{0}= & \eta_{2}\left\{L_{-2}+b^{+} \sqrt{G_{0}-b^{+} b+2 \mathcal{P}_{-2} \eta_{2}+2 \eta_{-2} \mathcal{P}_{2}}\right\}+ \\
& \eta_{-2}\left\{L_{2}+\sqrt{G_{0}-b^{+} b+2 \mathcal{P}_{-2} \eta_{2}+2 \eta_{-2} \mathcal{P}_{2}} b\right\}
\end{aligned}
$$

the inclusion of the constraints $L_{0}, L_{1}$ and $L_{-1}$ into the BRST charge is trivial. After the corresponding gauge fixing and solving the equations of motion for some of the auxiliary fields, one obtains the BRST quantized lagrangian, given in [10].

\section{Conclusions}

In this paper we have demonstrated the various constructions of the nilpotent BRST charges for a given algebra of constraints. The identification of 
the generators of the algebra with the constraints on the physical states is model-dependent and therefore after the quantization these BRST charges lead to the different spectrum of the physical states.

Acknowledgments. This investigation has been supported in part by the Russian Foundation of Fundamental Research, grants 96-02-17634 and 96-0218126, joint grant RFFR-DFG 96-02-00180G, and INTAS, grants 93-127-ext, 96-0308, 96-0538, 94-2317 and grant of the Dutch NWO organization.

\section{References}

[1] S.Ouvry, J.Stern. Phys.Lett., B177 (1986) 335

[2] J.M.F.Labastida. Phys.Rev.Lett., 58 (1987) 531

[3] J.M.F.Labastida. Nucl.Phys., B322 (1989) 185

[4] A. Pashnev, M. Tsulaia. Mod.Phys.Lett., A12 (1997) 861

[5] L.D. Faddeev, S.L. Shatashvili. Phys.Lett., B167 (1986) 225

[6] I.A. Batalin, E.S. Fradkin. Nucl.Phys., B279 (1987) 514

[7] E.T. Egoryan, R.P. Manvelyan. Theor. Math.Phys., 94 (1993) 241

[8] A. Pashnev, M. Tsulaia. Mod.Phys.Lett., A13 (1998) 1853

[9] I.M.Gel'fand, M.L.Tsetlin. Dokl. Akad. Nauk SSSR., 71 (1950) 825; ibid 71 (1950) 1017

[10] C.Fronsdal. Phys.Rev., D18 (1978) 3624 